ojs.uv.es/index.php/qfilologia/index

Rebut: 04.07.2020. Acceptat: 03.08.2020

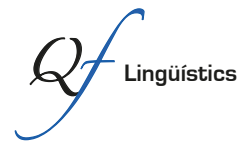

Per a citar aquest article: Meisnitzer, Benjamin. 2020. "Interfaces entre didáctica y lingüística:

las variedades europea y brasileña del portugués en la didáctica del portugués como lengua extranjera y la competencia variacional receptiva". Quaderns de Filologia: Estudis Lingüístics XXV: I85-20I.

doi: $10.7203 /$ Q.25.19079

\title{
Interfaces entre didáctica y lingüística: las variedades europea y brasileña del portugués en la didáctica del portugués como lengua extranjera y la competencia variacional receptiva
}

\author{
Interfaces between didactics and linguistics: European and Brazilian Portuguese, \\ foreign language teaching and multi-varietal passive competence
}

\author{
BenjAMin MeisnitZeR \\ Universidad de Leipzig \\ benjamin.meisnitzer@uni-leipzig.de
}

Resumen: El portugués brasileño (BP) y el europeo (EP) son ejemplos prototípicos de una lengua pluricéntrica, mientras que el caso de las distintas variedades africanas es más discutible. Las variedades lingüísticas son un desafío para la didáctica del portugués como lengua extranjera (L2 o L3) y lengua de herencia. Por ello, la presente contribución pretende discutir las ventajas de un acercamiento a la lengua extranjera (LE) a través de la enseñanza de una sola variedad o de aproximación pluricéntrica. En un segundo paso, presentamos algunas características relevantes para la adquisición de la competencia receptiva de la variedad brasileña en la enseñanza del portugués como LE, proponiendo también posibles vías didácticas para esta. El objetivo es la formación de hablantes de portugués L2 o L3 que tengan un buen dominio hablado y escrito en distintos contextos comunicativos y tradiciones discursivas.

Palabras claves: variedades; lengua extranjera; pluricentrismo; didáctica; competencia variacional.

Abstract: Brazilian (BP) and European Portuguese (EP) are prototypical examples of a pluricen-
tric language, while the status of African vernacular Portuguese varieties is still controversial.
The existence of different standard varieties is an enormous challenge for teaching Portuguese
as a foreign language, since it is hard to decide which variety to teach. Scholars are divided as
to whether a pluricentric approach or the focus on one variety only is the more fruitful way to
learn a foreign language. Based on results from recent studies on BP, we first present main dif-
ferences between EP and BP on different linguistic levels. In a further step, we propose some
practical approaches for including linguistic variation in classes for Portuguese as heritage
language, L2 or L3, without taking the risk of teaching an interlanguage based on the mixture
of both varieties (e.g. use of clitics). This reflection and approach is crucial to provide speaking 
and writing proficiency to learners of Portuguese as a foreign language with genuine proficiency for different discourse traditions and communicative contexts.

Keywords: Varieties; Portuguese as a Foreign Language; Pluricentric Languages; Didactics; Multi-Varietal Competence.

\section{El portugués como lengua pluricéntrica}

Si miramos las variedades brasileña y europea, el portugués es el prototipo de una lengua pluricéntrica. Partiendo de la definición sociolingüística de Clyne (I992), se puede definir una lengua pluricéntrica como lengua con "several interacting centres, each providing a national variety with at least some of its own (codified) norms" (Clyne, I992: 4). El caso del pluricentrismo del español, por ejemplo, se presenta de una forma bastante más controvertida. Esto se debe a la contingencia geográfica de las (posibles) distintas variedades estándar en América Latina y la dificultad en demarcarlas. Queda, además, abierta la cuestión de cuántas variedades del español se pueden considerar estándares (Bierbach, 2000; Oesterreicher, 200I; RAE, 20I8, VI-VII, etc.). Mientras Bierbach (2000) propone variedades nacionales, Oesterreicher (200I) distingue entre variedades regionales, cuyas isoglosas (léxicas, fónicas y morfosintácticas) no coinciden (necesariamente) con fronteras nacionales. Volviendo ahora al caso del portugués -y excluyendo las variedades africanas, menos consolidadas- observamos la existencia de dos espacios geográficos variacionales distintos con fronteras claras e isoglosas bien definidas. Ambos corresponden a variedades nacionales claramente identificables, las cuales sirven de referencia para las respectivas marcaciones diasistemáticas (diatópica, diastrática y diafásica ${ }^{\mathrm{I}}$ (Oesterreicher, 200I: 306). Así, para el portugués europeo el centro normativo de la variedad de la distancia comunicativa se localiza en el eje Lisboa-Coimbra, mientras que para el Brasil es el que va desde los centros urbanos de Rio de Janeiro y São Paulo (Arden \& Meisnitzer, 20I3: 2I) (Koch \& Oesterreicher, 2007: 26-35).

\footnotetext{
${ }^{\mathrm{I}}$ La variación diatópica hace referencia a las diferencias desde el punto de vista espacial (por ejemplo, las variedades dialectales como el dialecto extremeño o murciano). La variación diastrática concierne a las diferencias lingüísticas que se corresponden con la pertenencia a grupos y estratos sociales (los sociolectos) y la variación diafásica tiene que ver con los estilos de lengua que se adecuan a situaciones comunicativas (por ejemplo, español coloquial) (Koch \& Oesterreicher, 2007: 36-37).

${ }^{2}$ Koch \& Oesterreicher (I985, 2007 \& 20II), además de distinguir entre la lengua hablada y la lengua escrita en lo que concierne al medio, defienden la necesidad de distinguir entre oralidad
} 
La definición de lengua pluricéntrica de Clyne (véase arriba) es todavía demasiado vaga para la diversidad y complejidad de constelaciones pluricéntricas. Importa por eso añadir lo siguiente: (I) la relevancia de centros urbanos con carácter de modelo lingüístico para la variedad formal de la distancia comunicativa (Bierbach, 2000: I44); (2) consciencia lingüística y una identidad colectiva que permita que los hablantes de la variedad y los de otras variedades de la misma lengua histórica la reconozcan e identifiquen con un determinado espacio geográfico (Bierbach, 2000: I45-I47) y (3) que esa variedad sirva de modelo en la escuela, en los medios de comunicación y en las tradiciones discursivas de la distancia comunicativa (Bierbach, 2000: I48). (4) Para eso la respectiva variedad necesita de instrumentos codificadores (diccionarios, gramáticas prescriptivas y manuales ortográficos) (Bierbach, 2000, I46-I48). Además, en oposición a lo defendido por Bierbach (2000), nos vemos ante el problema de que las variedades estándar no necesariamente coinciden con las fronteras de los Estados nacionales. Por lo menos en algunos casos, lo que parece más determinativo es la existencia de centros urbanos, las megalópolis, cuya variedad sirve de referencia más allá de las fronteras nacionales (por ejemplo, Ciudad de México, Lima y Buenos Aires) y que por eso forman los llamados estándares regionales o zonales (Oesterreicher, 200I: 310). Además, Oesterreicher (200I: 3I0) enfoca la relevancia de la dimensión descriptiva, o sea, considera que las características lingüísticas con valor de referencia para aspectos lingüísticos marcados en las variedades del respectivo diasistema son más importantes que la consciencia lingüística de los hablantes. Cabe relativizar un poco este último punto, puesto que la consciencia lingüística de los hablantes es fundamental para el desarrollo de una variedad estándar autónoma, puesto que los instrumentos lingüísticos normativos y prescriptivos resultan de una emancipación de una variedad y del desarrollo de la consciencia de las particularidades lingüísticas de una variedad por parte de los hablantes (Krefeld \& Pustka, 20IO: I2-I5).

En el caso del portugués, se pueden distinguir diversas normas. Así, tenemos una norma endógena en Angola, el país africano con más hablantes del portugués como Li y con un número creciente de lusohablantes, sobre todo entre las generaciones más jóvenes (Castro, 2006: 32-33; Endruschat, 2008: 87). Mozambique es otro país con una tendencia significativa al desarrollo de una norma endógena, aunque en I997 todavía solo el 6 \% de la población

y escrituralidad en un nivel concepcional en lo que respecta a las tradiciones discursivas inmediatas y las distantes, respectivamente (Koch \& Oesterreicher, 2007: 35). 
indicaba el portugués como Li (Firmino, 2005: 6I3). Otro país en el que el portugués actualmente se encuentra en expansión es Timor Leste, donde en 2010 solo 595 de los habitantes indicaban hablar el idioma como LI, mientras que 449.085 indicaban el tetun (Meisnitzer, 2019: 23). Aun así, con el reconocimiento del portugués como lengua oficial y con su inclusión en los currículos escolares es de esperar que el número de hablantes aumente en los próximos años, aunque la euforia inicial de los años de la independencia se haya desvanecido (Cardeira, 2006: 88). Por lo general, el portugués está perdiendo su influencia en Asia -excepto en Timor Leste-, y ya solo se utiliza en la proximidad comunicativa (Meisnitzer, 2016).

En el caso del portugués brasileño, tenemos una variedad extraeuropea con su propio estándar, cuyo radio coincide con las fronteras nacionales. Las dos variedades se han desarrollado a partir de una misma lengua histórica (Teyssier, 200I: 74-78), pero por motivos sociales e históricos, entre los cuales cabe destacar la descolonización e independencia en el siglo xix, la variedad brasileña ha seguido su propio rumbo (Meisnitzer, 2019: 20). El conjunto de variantes que forman la variedad del portugués brasileño se extiende a todos los niveles lingüísticos. Es de destacar que actualmente se da una casi total ausencia de interferencias del portugués europeo en la variedad brasileña (Pöll, 20ı2: 35), lo que demuestra el alto grado de emancipación de la variedad emergente. Así, la proclisis, la forma del progresivo con gerundio y la palatalización de las oclusivas dentales delante de las vocales $<\mathrm{e}>\mathrm{e}<\mathrm{i}>$ son apenas algunos de los ejemplos más prominentes neutrales del punto de vista de la marcación diasistemática en Brasil, que divergen de otras variedades estándar (nacionales) del portugués. En Brasil, estos elementos lingüísticos están integrados en la norma hablada culta y sirven de referencia desde una perspectiva gramaticográfica y lexicográfica [véase, por ejemplo, la Nova Gramática do Português Brasileiro de Ataliba de Castilho (20I0), la Gramática Pedagógica do Português Brasileiro de Marcos Bagno (2012) o el diccionario del portugués brasileño Dicionário Houaiss da Língua Portuguesa (2009)]. Esta norma se refleja también en la enseñanza del portugués en Brasil. Un papel decisivo en el desarrollo de las variedades del portugués europeo y brasileño en la actualidad desempeñan los medios de comunicación social, sobre todo, la televisión (Mateus \& Cardeira, 2007: 39). Evidentemente, estos medios no inician de forma consciente los procesos de cambio lingüístico, pero sí determinan la consolidación de las "nuevas" normas endógenas, ya que facilitan su difusión (Meisnitzer, 2019: 22). 
Con respecto al campo de la enseñanza del portugués como lengua extranjera o lengua de herencia, la cuestión del pluricentrismo y de sus posibles implicaciones sigue siendo marginal y poco discutida. Eso es problemático: por una parte, se intenta impartir clases de las dos variedades en las universidades. Por otra, no se logra ofrecer los espacios didácticos necesarios para reflexionar las convergencias y las divergencias entre los diferentes estándares.

\section{Didáctica de lenguas pluricéntricas como lengua extranjera}

Desde el punto de vista de la lingüística, la cuestión de si la enseñanza debe partir de una sola variedad o tomar un rumbo pluricéntrico parece fácil de contestar, puesto que la lingüística se interesa por la variación y las variedades lingüísticas. En el campo de la didáctica de las lenguas extranjeras, sin embargo, el asunto es mucho más controvertido (Leitzke-Ungerer, 20I7; Reimann, 20I7). Para los estudiantes de lengua extranjera, la adquisición de las reglas en sí ya no es fácil y, por tanto, la confrontación con varias variedades de una lengua pluricéntrica puede llevar a una adquisición deficiente del idioma, en el sentido de que el aprendiente acaba adquiriendo una "intervariedad". Este riesgo es mayor cuanto más cerca están las variedades entre sí. El portugués es, precisamente, una lengua de este tipo: a pesar de las diferencias entre el PE y el PB (sección 3), ambas coinciden en un gran número de aspectos léxicos y morfosintácticos. Además, la variedad portuguesa enseñada en las escuelas brasileñas como norma lingüística no se ha alejado tanto de la variedad europea, como haría suponer, sobre todo, quizá el aspecto fónico . En esta contribución, se defiende la perspectiva de que la variación lingüística debe formar parte del proceso de aprendizaje del portugués como lengua extranjera, pero solo en niveles avanzados, cuando los conocimientos básicos ya estén consolidados. Esto no solo es importante desde una perspectiva puramente lingüística, sino también ante el discurso sociolingüístico brasileño: algunos gramáticos brasileños, tal como Marcos Bagno (2012: 202 y 253), ya reivindican la designación del idioma como "brasileño" y no "portugués brasileño".

\subsection{Variedades del portugués en los manuales de PLE}

Los manuales de portugués como lengua extranjera (PLE) editados en Brasil no consideran otra variedad que la brasileña, mientras que en los manuales portugueses hay por lo menos una cierta tendencia a dar cuenta de caracterís- 
ticas del PB a través de textos y ejercicios de comprensión oral. Solo los manuales Português XXI, volumenes 2 y 3 , intentan dar cuenta de las particularidades del portugués brasileño y angolano, por mucho que la presentación de las especificidades lingüísticas sea a veces problemática. Johnen (20I0: 97), por ejemplo, destaca el hecho de que muchos de los fenómenos lingüísticos abordados (a) ocurren en ambas variedades, pero (b) tienen valores diasistemáticos muy distintos, lo cual (c) no se menciona.

En los manuales editados en países no lusohablantes, se observa una presencia más significativa de la variación geolingüística. Estos manuales adoptan el PE o el PB como variedad de referencia y explican los rasgos lingüísticos de la otra variedad desde un punto de vista contrastivo. Un intento en ese sentido constituye Ponto de Encontro (Jouët-Pastré et al. 2013), en el que se presentan en cada unidad textos para una misma situación comunicativa en PB y en PE (en este orden). Sin embargo, como destaca Johnen (2019: 96), los fenómenos lingüísticos introducidos son los más conocidos y no van acompañados de ninguna reflexión metalingüística con respecto a su utilización en la otra variedad. Por lo tanto, esto puede resultar en supergeneralizaciones por parte de los aprendices. Según Johnen (2019: 97), un manual que intenta minimizar este riesgo es Boléo \& Prata \& Silva (20I7), el cual ha sido redactado por autores provenientes de diferentes países lusófonos. Sin embargo, ni los textos ni los audios son auténticos en este manual.

Ahora, el desafío de la didáctica de las lenguas extranjeras no solo consiste en dar cuenta de la variación lingüística, sino también en evitar que esta cause interferencias en la consolidación de la variedad estudiada por el aprendiz. Un ejemplo muy evidente es el de la colocación de los clíticos. En el PB domina la proclisis, mientras que en el PE domina la enclisis en oraciones no marcadas. Sin embargo, esta generalización no es suficiente pare hacer justicia a la complejidad de los usos de los clíticos, que en PE se utilizan de forma proclítica en oraciones negativas (I), en oraciones subordinadas (2), en oraciones interrogativas o exclamativas introducidas por un pronombre interrogativo (3), en oraciones con pronombres indefinidos con función de sujeto (4) y con algunos adverbios focalizados y focalizadores (5).

(I) Não lhe deu o dinheiro.

(2) Creio que lhe deu o dinheiro.

(3) Porque lhe deu o dinheiro?

(4) Ninguém nos ajudou. 
(5) Ainda se lembra desses bons tempos?

(Schäfer-Prieß \& Schöntag, 20I2: I48)

Estos ejemplos ponen de manifiesto el riesgo de supergeneralización por parte de los aprendices, destacado por Johnen (2019; véase arriba). Sin sensibilizar a los aprendices con respecto a la verdadera complejidad de la utilización de los clíticos (p. ej., con reglas vagas como "El PB prefiere la proclisis y el PE la enclisis"), acabarán adquiriendo una variedad que no se corresponderá ni al PE ni al PB. Lo mismo sucederá si los manuales destacan que en el $\mathrm{PE}$ los posesivos van precedidos de artículo definido sin tampoco mencionar que en PB también existen contextos en los que se utiliza este patrón (Johnen 2019: 96). Otro problema, por supuesto, es el posible cambio de enseñantes. Si estos cambian y los aprendices pasan de ser instruidos por docentes hablantes de PE a docentes nativos de PB, se corre de nuevo el riesgo de adquisición de una intervariedad.

\subsection{Variedades del portugués en la didáctica del PLE: ¿qué variedad(es) enseñar?}

Una discusión central en la didáctica de las lenguas extranjeras pluricéntricas es siempre la cuestión de qué variedad enseñar. En el caso del portugués, se han defendido enfoques muy divergentes. En Alemania, los cursos de portugués lengua de herencia utilizan sobre todo el PE, debido a las olas migratorias de portugueses hacia este país. En los cursos de PLE en los Centros de Idiomas (Sprachenzentren) de las universidades, con sus cursos para aprendientes que no son de Filología, se enseña, sin embargo, más bien el PB (por ejemplo, en las Universidades de Leipzig o Zúrich3). Los cursos impartidos en los institutos universitarios de Filología, en cambio, son, por lo general, de PE. Finalmente, también existen currículos universitarios que ofrecen a los estudiantes de portugués (a) escoger entre cursos en una de las dos variedades (Colonia) o (b) cursar cursos de lengua adicionales que abordan, en un nivel de aprendizaje medio-avanzado, el PB (Múnich). El panorama de los cursos de portugués LE es bastante heterogéneo en lo que concierne a la variedad enseñada y a la inclusión de la otra variedad en el currículo, lo que

\footnotetext{
${ }^{3}$ Estos datos corresponden a una consulta de las páginas web de los respectivos institutos hecha en 15 de enero de 2020.
} 
desde el punto de vista didáctico es problemático, sobre todo en tiempos de movilidad de los estudiantes, quienes muchas veces cambian de universidad durante su carrera.

La variedad enseñada en los niveles iniciales de una lengua extranjera debe ser la de los manuales, que, por ejemplo, en el caso de la enseñanza del español como LE en Alemania es la variedad europea (Leitzke-Ungerer, 20I7: 65). El uso de una variedad distinta a la del manual de clase, en cambio, retrasa el proceso de aprendizaje. Esto es debido a las diferencias lingüísticas -fónicas, morfológicas, sintácticas, pragmáticas o semánticas-, las cuales causan interferencias que, a su vez, dificultan la consolidación y la adquisición de las reglas por parte de los estudiantes (piénsese, por ejemplo, en la colocación de los clíticos en PE y PB). Aun así, en el caso del portugués, la situación no está tan clara, puesto que se observa la coexistencia de numerosos manuales portugueses y numerosos manuales brasileños (por ejemplo, Novo Avenida Brasil de la editorial E.P.U. o Fala Brasil de la editorial Pontes). Otro aspecto decisivo puede ser el de la inmersión de los aprendices en la lengua extranjera, que en la mayoría de los casos ocurre en el semestre de Erasmus en Portugal, por lo que la variedad portuguesa sería la ideal. Si, por el contrario, tenemos en cuenta argumentos fonético-fonológicos y la facilidad de comprensión y la proximidad entre pronunciación y escritura, tendríamos que optar por el PB en las clases de portugués como LE. El problema si no hay continuidad varietal en los primeros niveles (hasta el nivel B2) es el riesgo de inseguridad de los aprendices debido a la diversidad de formas y la subsiguiente producción de errores, que ponen en riesgo de forma sustancial la adquisición de bases lingüísticas en la LE (Leitzke-Ungerer, 20I7: 65). Esto es sobre todo válido para los primeros años en los que los aprendientes adquieren la pronunciación y las formas de tratamiento, áreas en las que las dos variedades presentan diferencias muy profundas. En los demás dominios lingüísticos, es importante fomentar la competencia productiva en una variedad estándar y competencia perceptiva en la otra variedad (e incluso en niveles más avanzados en las otras variedades del portugués, así como, por ejemplo, la angolana). Para ello, algunos manuales ofrecen textos o audios que pueden usarse (por ejemplo, Boléo $\&$ Prata \& Silva, 20I7).

Ante este problema, opinamos que quizás sería mejor que los profesores recurrieran a materiales auténticos, como pasajes de libros infantiles o periódicos, o incluso a materiales audio tomados de reportajes o entrevistas (para ejemplos, véase Meisnitzer, 2019: 35-39). 
Una vez consolidados los conocimientos básicos en una de las variedades, se deben dar a conocer sucesivamente las características de la(s) otra(s) variedad(es) para transmitir a los aprendientes competencias inter- y transculturales, de importancia central en un mundo cada vez más globalizado y conectado (Reimann, 20II: I25-I28). Según este modelo didáctico, el que Reimann denomina de didáctica pluricéntrica, el objetivo es que los aprendientes sean capaces de identificar otra(s) variedad(es) por sus características lingüísticas más salientes (competencia variacional receptiva) (Reimann, 20I7: 72-73; Reimann \& Koch, 20I9: IO-II).

La necesidad de desarrollar una competencia comunicativa transcultural exige que se desarrolle una didáctica y métodos de enseñanza para el portugués lengua pluricéntrica. En el caso del portugués, la enseñanza se puede limitar a las variedades europea y brasileña, puesto que las variedades africanas todavía no están lo suficientemente consolidadas para la elaboración de manuales. Muchos de los aspectos lingüísticos "característicos" de estas últimas variedades son todavía muy inestables, lo cual se puede explicar por la implementación relativamente tardía del portugués en los países africanos de lengua oficial portuguesa (PALOP), independientes solo desde I975.

Como en los cursos de portugués LE en Alemania, Austria y Suiza la variedad más enseñada sigue siendo el PE, en el siguiente apartado proponemos características lingüísticas del PB que deben integrarse en un currículo de competencia receptiva.

\section{Características lingüísticas del PB que deben formar parte de la adquisición de una competencia receptiva para aprendientes del PE}

Como ya se ha mencionado en el apartado I, Brasil está caracterizado por una enorme discrepancia entre la norma prescriptiva, que se acerca mucho al estándar del PE, y las normas cultas habladas, las cuales sirven de base para la norma descriptiva de las tradiciones discursivas y situaciones comunicativas de la distancia comunicativa del PB. Estas hablas cultas, a su vez, se distancian mucho de las hablas populares, que corresponden al habla de las clases sociales con un bajo grado de instrucción o incluso iletrados. Lo que es importante para la didáctica es distinguir entre formas lingüísticas que forman parte de las normas cultas populares y las que pertenecen a las normas populares habladas. Las primeras constituyen el grupo de las características lingüísticas que pueden -y deben- enseñarse a los aprendientes del PE como 
rasgos típicos del PB. Las últimas, en cambio, son rasgos lingüísticos diastrática y diafásicamente bajos en el sentido de Koch \& Oesterreicher (2007: 39). En contraposición a los rasgos lingüísticos del primer grupo, estos últimos no forman parte de los registros cultos de las variedades estándar, razón por la cual se desaconseja incluirlas en la enseñanza del PLE.

BP remains in an area of conflict and tension between spoken speech and written language, both with their own competing norms. There is still a lot of work to be done on the distinction between marked and unmarked elements within the diasystem of BP and on the description and discussion of non-dominant features becoming dominant. (Martins \& Meisnitzer, 20I6: 69)

\subsection{Características fonéticas y fonológicas del PB}

En el nivel fonético y fonológico, una de las diferencias más marcadas entre el PE y el PB es la palatalización de las consonantes oclusivas / t/ y /d/ cuando están seguidas de vocal [i] o de semivocal [j] (por ejemplo, en tio ['tfiu] y dente ['dẽtfi], nótese también el cierre anterior de [e] a [i], o ['tfjagu]) (Mattos e Silva, 20I3: I50). En la palabra dente, en la sílaba átona en el fin de la palabra, en el PE tenemos una centralización y una elevación de la vocal [i] (Mattos e Silva, 2013: I49).

Una característica muy marcada del consonantismo del PE es la palatalización de la consonante fricativa coronal /s/ en posición final de palabra (ej. - cai[J]), en final de sílaba y antes de consonante (ej.- po[J]to), que en Brasil solo ocurre en el habla culta de Río de Janeiro (Mattos e Silva, 20I3: I50).

Además, el PB se caracteriza por el debilitamiento de la consonante lateral /1/ en la coda silábica, realizada como semivocal [w] (por ejemplo, ma[w]dade) y por la pérdida de la consonante vibrante / $\mathrm{r} /$ en la coda ( $\operatorname{am}[\mathrm{a}]$ ) (Mattos e Silva, 20I3: I50). En dos contextos -antes de una pausa o de una consonante- la vibrante se realiza como fricativa glotal $(a m a[h])$ o como fricativa velar ( $a m a[\mathrm{x}])$ (Mattos e Silva, 20I3: I50). Cuando tenemos la consonante vibrante en posición final de palabra y antes de una palabra iniciada por vocal, se realiza como coronal simple a semejanza del PE (ama[r]) (Mattos e Silva, 20I3: I50).

En el campo del vocalismo del PE es de destacar la elevación y/o centralización de las vocales átonas de una forma categórica, lo que no ocurre en Brasil (tabla I). 


\begin{tabular}{|c|c|}
\hline \multicolumn{2}{|c|}{ Sílaba pretónica } \\
\hline PB & $P E$ \\
\hline$p[0] r t a l$ & $p[u] r t a l$ \\
\hline I[e]vada & [[i]vada \\
\hline$c[a] r t a d a$ & $c[\mathrm{e}]$ rtada \\
\hline I[o]bão & I[u]bão \\
\hline$p[e] r e i r a$ & $p[i]$ reira \\
\hline \multicolumn{2}{|c|}{$m[i] / h e i r o, c r[u] z e i r o$} \\
\hline \multicolumn{2}{|c|}{ Sílaba átona, posición final de palabra } \\
\hline $\operatorname{lev}[\mathrm{i}]$ & $\operatorname{lev}[\dot{]}],[[\varepsilon v]$ \\
\hline \multicolumn{2}{|c|}{$\operatorname{terr}[\mathrm{e}], \operatorname{cert}[\mathrm{u}]$} \\
\hline
\end{tabular}

Tabla I. Diferencias en lo que concierne al timbre de vocales átonas entre el PB y el PE (adaptado de Mattos e Silva, 20I3: I49)

Las vocales tónicas son idénticas en el PB y el PE.

\subsection{Características morfosintácticas del PB}

En la enseñanza del portugués como LE, para un tratamiento de características lingüísticas del PB, es importante subrayar las diferencias entre el lenguaje hablado de la distancia comunicativa (habla formal o culta) y la norma padrão prescriptiva de la lengua escrita (véase la tabla 2).

Los brasileños con un nivel académico elevado utilizan los rasgos lingüísticos correspondientes a las normas del habla culta. La norma padrão, que es en realidad una norma exógena de Portugal, es todavía -pero exclusivamente- la norma vigente en los exámenes de acceso a la universidad (vestibular). Esta norma del discurso escrito está ya obsoleta entre los hablantes en la lengua hablada, incluso en situaciones comunicativas formales, y se aleja enormemente de lo que utilizan en lo escrito o lo hablado en las situaciones de su vida cotidiana (incluso los hablantes con formación académica; Duarte, 20I2). Esta tensión entre lo hablado y lo escrito está llevando a un proceso de reorganización de la gramática del $\mathrm{PB}$, un proceso que todavía no está concluido. Sin embargo -y teniendo en cuenta el principio comunicativo de la didáctica PLE vigente-, es importante enseñar estos rasgos lingüísticos, una vez que forman parte de la variedad de referencia para los hablantes brasileños. 
More recently, however, [...] there has been a move, especially among mass media, towards decreasing the gap between spoken and written BP so as to reach the audience/the readers more efficiently. (Duarte \& Gomes \& Paiva, 20I6: 62)

E incluso las gramáticas del PB escrito tienden a incluir cada vez más los rasgos lingüísticos endógenos brasileños (de la habla culta) (Duarte \& Gomes \& Paiva, 2016: 63).

\begin{tabular}{|c|l|l|}
\hline & \multicolumn{1}{|c|}{ Norma (padrão) PB } & \multicolumn{1}{|c|}{ Habla culta PB } \\
\hline 1. & Deu-me dinheiro para comprar roupa. & Me deu dinheiro para comprar roupas. \\
\hline 2. & $\begin{array}{l}\text { Vi-o na rua. } \\
\text { Vou comprá-los }\end{array}$ & $\begin{array}{l}\text { Vi ele na rua. } \\
\text { Vou comprar } \varnothing . / \text { Vou comprar eles. }\end{array}$ \\
\hline 3. & $\begin{array}{l}\text { Deixa-me dizer o que penso disso. } \\
\text { Vi-o sair da sala. }\end{array}$ & $\begin{array}{l}\text { Deixa eu dizer o que penso disso. } \\
\text { Vi ele sair da sala. }\end{array}$ \\
\hline 4. & $\begin{array}{l}\text { Fui ao festival. } \\
\text { Cheguei à estação atrasado. }\end{array}$ & $\begin{array}{l}\text { Fui no festival. } \\
\text { Cheguei na estação atrasado. }\end{array}$ \\
\hline 5. & $\begin{array}{l}\text { Esse é um livro de que eu gosto muito. } \\
\text { Essa é a vizinha em cuja casa jantei } \\
\text { ontem. }\end{array}$ & $\begin{array}{l}\text { Esse é um livro Ø que eu gosto muito. } \\
\text { Essa é a vizinha Ø que eu jantei na casa } \\
\text { ontem. }\end{array}$ \\
\hline
\end{tabular}

Tabla 2. Características del habla culta brasileña en comparación con la norma patrón de la lengua escrita (Arden, 2010: 7)

En el PB, la proclisis está más generalizada que en el PE e incluso puede ocurrir en posición inicial de oración con los clíticos me, te y se (cf. I, tabla 2), lo que la norma escrita no permite ${ }^{4}$. Además, la mesoclisis del PE se transforma en proclisis (PE dar-me-á > PB me dará). Mientras que la norma prescriptiva del PB pide objetos directos clíticos, las hablas cultas los remplazan por objetos nulos o por pronombres de sujeto (cf. 2, tabla 2). Los verbos mandar, fazer, deixar, ver, ouvir y sentir seguidos de infinitivo piden el uso de un clítico de COD; en el habla culta, los hablantes utilizan la forma nominativa de los pronombres (cf. 3, tabla 2). Los verbos de movimiento ir y chegar requieren la preposición en en el habla culta, mientras que el uso patrón pide la preposición a para introducir el complemento de lugar (cf. 4, tabla 2). Finalmente, las variedades cultas habladas omiten las preposiciones delante de pronombres relativos (cf. 5, tabla 2) y generalizan el pronombre você para la segunda per-

\footnotetext{
${ }^{4}$ En textos de periodistas o estudiantes se puede notar una tendencia hacia el uso de la enclisis incluso en contextos en los que el PE exige la proclisis (hipercorrección): O vestido que dei-lhe de presente ficou bom. (Mattos e Silva, 20I3: 153).
} 
sona del singular (6), forma estigmatizada en PE, puesto que se trata de una forma marcada diastráticamente en su uso generalizado. En PE, você solo lo puede utilizar un superior para dirigirse a su empleado o entre colegas.

(6) Você gosta mesmo de futebol!

Digna de mención es también la pérdida de lhe como complemento indirecto y su uso como complemento directo en variedades dialectales (7). Lo mismo ocurre con el uso del pronombre de reanudación en oraciones relativas (pronome de retoma o lembrete) (8).

(7) Você gosta mesmo de golfe! Eu lhe vejo sempre no clube. (Mattos e Silva, 20I3: I52)

(8) O cão que eu gostei dele fugiu. (Mattos e Silva, 2013: I53)

Es importante identificar estos fenómenos (cf. 7 \& 8) como tendencias de las normas populares habladas, que no deben ser enseñadas en las clases de LE, puesto que se trata de formas diastráticamente marcadas. Lo mismo ocurre con la reducción del paradigma verbal de cuatro formas en la variedad hablada culta (eu falo; você, ele/ela, a gente fala ([nós] falamos); vocês, eles/elas falam) hacia dos formas (eu falo; você, ele/ela, a gente, vocês fala) en la variedad hablada popular (Arden, 20I0: 9). O la ausencia de marcación de la concordancia de número en el sintagma nominal en la variedad hablada popular (os nosso_amigo_) (Mattos e Silva, 2013: 151).

Puesto que en el PB actual se registran procesos de cambio lingüístico que afectan a estructuras, fenómenos lingüísticos y estrategias discursivas bajo la influencia del habla culta, estos son aspectos que se deben incluir en los manuales de portugués LE para luego poder desarrollar en los aprendices una competencia receptiva de tales variedades.

\subsection{Recomendaciones para la adquisición de una competencia receptiva del $P B$}

Hemos visto que lo importante es que los aprendices sean capaces de identificar y de entender el PB (competencia pasiva). Para ello, se debe utilizar material audiovisual o audios auténticos, como secuencias de noticiarios televisivos como el Jornal Nacional. La secuencia en el ejemplo (8) se podría 
utilizar para explicar el uso de la proclisis (I), divergencias en la regencia de preposiciones con complementos circunstanciales de lugar (2) (PE: à), la mayor productividad del condicional en el PB y diferencias en su utilización entre el PB y el PE $(4)^{5}$ y diferencias en las perífrasis verbales (5) (acabar saindo en vez de acabar por sair (PE)), discutiendo aquí también el contraste de la forma progresiva, formada con el gerundio en PB y con infinitivo en PE. (8)

C. Carvalhar, nutricionista - A pessoa se (I) serve muito, lá na (2) frente acha uma coisa Ø (3) que gostaria (4) mais, coloca e acaba saindo (5) do restaurante empanturrada (JN, I5/10/2008 apud Arden, 2015: 254).

El fenómeno lingüístico quizás más llamativo es la omisión de preposiciones a la hora de introducir oraciones relativas oblicuas (relativa cortadora) (3), un proceso muy productivo en el PB hablado. Las secuencias de noticiarios televisivos cuenta con la ventaja de que se trata de formatos de fácil acceso, de fácil transcripción y con suporte audiovisual, lo que facilita la comprensión y motiva a los aprendices. Lo importante es, obviamente, la adaptación de los materiales conforme el nivel de los aprendices ${ }^{6}$.

\section{Conclusión: didáctica del portugués lengua pluricéntrica como LE}

En la didáctica de las lenguas extranjeras moderna, domina la opinión de que se debe transmitir a los aprendices competencias productivas en una variedad y competencias receptivas en las demás variedades (en el caso de las lenguas pluricéntricas). Por lo tanto, las características del PB presentadas tienen que formar parte de un material didáctico (auténtico) utilizado en cursos para aprendices del $\mathrm{PE}^{7}$, para así asegurar la adquisición de una competencia receptiva del PB.

Finalmente, también nos parece relevante añadir que, debido al pluricentrismo equilibrado entre PB y PE y debido a las diferencias profundas entre las dos variedades, siempre debería ser posible identificar directamente en la

\footnotetext{
${ }^{5}$ En (4), en el PE se utilizaría el presente por la consecutio temporum. Otra diferencia significativa es el uso del condicional en el PB para expresar cortesía y del imperfecto en el PE.

${ }^{6}$ Para un análisis más profundizado del ejemplo y más ejemplos de otras tradiciones discursivas, véase Meisnitzer (2019: 36-39).

7 En el caso del portugués se podría optar por el PE o el PB, como variedad enseñada y respectivamente transmitir competencias receptivas en la otra variedad. Lo importante siendo también, lo repetimos, que los docentes, sobre todo durante los primeros años, sean hablantes de la misma variedad.
} 
denominación de los cursos de PLE cuál de las dos variedades será la enseñada en el curso en cuestión.

\section{Bibliografía}

Arden, Mathias. 20I0. Zum Verhältnis von Gattungskonventionen und sprachlichen Normen in der Fernsehkommunikation - Telenovelas und Jornal Nacional von TV Globo. En Endruschat, Annette \& Kemmler, Rolf (ed.) Portugiesische Sprachwissenschaft: traditionell - modern - innovativ. Tübingen: Calepinus (Lusitanistische Sprachwissenschaft; 2), 5-32.

Arden, Mathias. 20I5. Inszenierte und elaborierte Mündlichkeit bei TV Globo. Zur soziostilistischen Modellierung morphosyntaktischer Variablen des brasilianischen Portugiesisch. Frankfurt am Main: Lang (Studia Romanica et Linguistica; 4I).

Arden, Mathias \& Meisnitzer, Benjamin. 20I3. Plurizentrik und massenmediale Normen: Der Fall des Portugiesischen. En Merlan, Aurélia \& Schmidt-Radefeldt, Jürgen (ed.) Das Portugiesische als Diasystem innerhalb und außerhalb des lusophonen Raums. Berlin: Lang, I9-52.

Bagno, Marcos. 20I2. Gramática Pedagógica do Português Brasileiro. São Paulo: Parábola.

Boléo, Maria João Manso \& Prata, Maria \& Silva, Alexandra da Fonseca. 20I7. Olá Portugal! Neu A1-A2: Portugiesisch für Anfänger. Kursbuch mit MP3-CD. Stuttgart: Klett.

Bierbach, Mechthild. 2000. Spanisch - eine plurizentrische Sprache? Zum Problem von norma culta und Varietät in der hispanophonen Welt. Vox Romanica 59: I43-I70.

Cardeira, Esperança. 2006. História do Português. Lisboa: Caminho.

Castilho, Ataliba Teixeira de. 20Io. Nova Gramática do Português Brasileiro. São Paulo: Contexto.

Castro, Ivo. 2006. Introdução à História do Português. 2. ${ }^{\mathrm{a}}$ ed.. Lisboa: Colibri.

Clyne, Michael. I992. Pluricentric Languages - Introduction. En Clyne, Michael (ed.) Pluricentric Languages. Differing Norms in Different Nations. Berlin, New York: de Gruyter, I-9.

Duarte, Maria Eugênia Lamoglia. When speech and writing are too far apart! Non-dominant features of Brazilian Portuguese becoming dominant. En Muhr, Rudolf (ed.): Non-Dominant Varieties of Pluricentric Languages. Getting the Picture. In Memory of Michael Clyne. Frankfurt am Main: Lang (Österreichisches Deutsch. Sprache der Gegenwart; I4), 315-326.

Duarte, Maria Eugênia L. \& Gomes, Christina Abreu \& Paiva, Maria da Conceição. 2016. "Codification and Standardisation in Brazilian Portuguese". En Muhr, Rudolf (ed.): Pluricentric Languages and Non-Dominant Varieties Worldwide. Part II: The Pluricentricity of Portuguese and Spanish. New Concepts and Descriptions. In collaboration with Eugênia Duarte, Amália Mendes, Carla Amorós 
Negre \& Juan Thomas. Frankfurt am Main: Lang (Österreichisches Deutsch. Sprache der Gegenwart; i9), 51-65.

Endruschat, Annette. 2008. Linguagem dos musseques - Die Stadtsprache Luandas. En Krefeld, Thomas (ed.) Sprachen und Sprechen im städtischen Raum. Frankfurt a. M.: Peter Lang, 77-IO2.

Firmino, Gregório. 2005. Línguas de Moçambique. En Cristóvão, Fernando \& Amorim, Maria Adelina \& Marques, Maria Lúcia Garcia \& Moita, Susana Brites (ed.) Dicionário Temático da Lusofonia. Lisboa: Texto. 6II-6I4.

Johnen, Thomas. 20I9. As variantes do português em manuais de Português Língua Estrangeira. En Reimann, Daniel \& Koch, Christian (ed.) As variedades do Português no Ensino de Português Língua Não Materna. Tübingen: Narr, Francke, Attempto (Romanistische Fremdsprachenforschung und Unterrichtsentwicklung; I4), 87-ıог.

Jouët-Pastré, Clémence \& Klobucka, Ana M. \& Sobral, Patrícia Isabel Santos \& Moreira, Maria Luci de Biaji \& Hutchinson, Amélia. 20I3. Ponto do Encontro. Portuguese as a world language. $2^{\mathrm{a}}$ ed. Boston: Pearson.

Koch, Peter \& Oesterreicher, Wulf. I985. Sprache der Nähe - Sprache der Distanz: Mündlichkeit und Schriftlichkeit im Spannungsfeld von Sprachtheorie und Sprachgeschichte. Romanistisches Jahrbuch 36(1985): I5 - 43.

Koch, Peter \& Oesterreicher, Wulf. 2007. Lengua Hablada en la Romania: Español, Francés, Italiano. Trad. y adaptación de Araceli López Serena. Madrid: Gredos (=Biblioteca Románica Hispánica. Estúdios y Ensaios; 448).

Koch, Peter \& Osterreicher, Wulf. 20II. Gesprochene Sprache in der Romania. Französisch, Italienisch, Spanisch. $2^{\text {a }}$ ed. New York/Berlin: de Gruyter (Romanistische Arbeitshefte; 3I).

Krefeld, Thomas \& Pustka, Elissa. 20ıо. Für eine perzeptive Varietätenlinguistik. En Krefeld, Thomas \& Pustka, Elissa (ed.) Perzeptive Varietätenlinguistik. Frankfurt a. M.: Lang (Kommunikative Räume/ Spazi Comunicativi; 8). 9-28.

Leitzke-Ungerer, Eva. 20I7. Vosotros oder ustedes: Wie viele Standardvarietäten verträgt der Spanischunterricht in den ersten Lehrjahren? En Leitzke-Ungerer, Eva \& Polzin-Haumann, Claudia (ed.) Varietäten des Spanischen im Fremdsprachenunterricht. Ihre Rolle in Schule, Hochschule, Lehrerbildung und Sprachzertifikaten. Stuttgart: Ibidem (Romanische Sprachen und ihre Didaktik; 6I), 4I-68.

Mateus, Maria Helena Mira \& Cardeira, Esperança. 2007. Norma e Variação. Lisboa: Caminho.

Martins, Marco Antonio \& Meisnitzer, Benjamin. 20I6. The use of clitics in Brazilian Portuguese - the development of an endogenous standard variety. En Muhr, Rudolf (ed.) Pluricentric Languages and Non-Dominant Varieties Worldwide. Part II: The Pluricentricity of Portuguese and Spanish. New Concepts and Descriptions. In collaboration with Eugênia Duarte, Amália Mendes, Carla Amorós Negre \& Juan Thomas. Frankfurt am Main: Lang (Österreichisches Deutsch. Sprache der Gegenwart; I9), 67-84.

Mattos e Silva, Rosa Virgínia. 20I3. O Português do Brasil. En Raposo, Eduardo Buzaglo Paiva; Bacelar do Nascimento, Maria Fernanda; Mota, Maria Antónia 
Coelho da; Segura, Luísa \& Mendes, Amália (ed.) Gramática do Português. Vol. I. Lisboa: Fundação Calouste Gulbenkian, I45-I54.

Meisnitzer, Benjamin. 20I6. Das Portugiesische in Asien als 'untergehende' Minderheitensprache des Nähebereichs. En Born, Joachim \& Ladilova, Anna (ed.) Sprachkontakte des Portugiesischen. Ausgewählte Beiträge der gleichnamigen des 10. Deutschen Lusitanistentages vom 10. - 14. September 2013 in Hamburg. Frankfurt: Lang (Iberolinguistica - Studien zur Sprach- und Kulturwissenschaft; 2), I47-169.

Meisnitzer, Benjamin. 20I9. O Português como língua pluricêntrica. Um desafio para a didáctica do Português Língua Estrangeira. En Reimann, Daniel \& Koch, Christian (ed.) As variedades do Português no Ensino de Português Língua Não Materna. Tübingen: Narr, Francke, Attempto (Romanistische Fremdsprachenforschung und Unterrichtsentwicklung; I4), I9-43.

Oesterreicher, Wulf. 200I. Plurizentrische Sprachkulturen - der Varietätenraum des Spanischen. Romanistisches Jahrbuch 5I(2000): 287-318.

Pöll, Bernhard. 20ı2. Situaciones pluricéntricas en comparación: El español frente a otras lenguas pluricéntricas. En Lebsanft, Franz; Mihatsch, Wiltrud \& Polzin-Haumann, Claudia (ed.) El español ¿desde las variedades a la lengua pluricéntrica? Frankfurt a.M.: Vervuert/Iberoamericana, 29-45.

RAE. 20I8. Nueva Gramática de la lengua española. Manual. 3. ${ }^{2}$ ed. Barcelona: Asociación de Academias de la Lengua Española.

Reimann, Daniel. 20II. Diatopische Varietäten des Französischen, Minderheitensprachen und Bilinguismus im transkulturellen Fremdsprachenunterricht. En Frings, Michael \& Schöpp, Frank (ed.) Varietäten im Französischunterricht. Stuttgart, Ibidem (Französischdidaktik im Dialog; I), I23-I68.

Reimann, Daniel. 20I7. Rezeptive Varietätenkompetenz: Modellierung einer Teilkompetenz zwischen funktionaler kommunikativer Kompetenz und Sprachbewusstsein. En Leitzke-Ungerer, Eva \& Polzin-Haumann, Claudia (ed.) Varietäten des Spanischen im Fremdsprachenunterricht. Ihre Rolle in Schule, Hochschule, Lehrerbildung und Sprachzertifikaten. Stuttgart: Ibidem (Romanische Sprachen und ihre Didaktik; 6r), 69-95.

Reimann, Daniel \& Koch, Christian. 20I9. O ensino de Português Língua Não Materna com enfoque na competência de variedades. A relevância das variedades na aula. En Reimann, Daniel \& Koch, Christian (ed.) As variedades do Português no Ensino de Português Língua Não Materna. Tübingen: Narr, Francke, Attempto (Romanistische Fremdsprachenforschung und Unterrichtsentwicklung; I4), 9-16.

Schäfer-Prieß, Barbara \& Schöntag, Roger. 20I2. Spanisch/Portugiesisch kontrastiv. Berlin, Boston: de Gruyter (Romanistische Arbeitshefte; 56).

Teyssier, Paul. 200 . A História da Língua Portuguesa. Lisboa: Livraria Sá da Costa. 
